УДК 511.3

\title{
Владимир Григорьевич Чирский (к 70-летию со дня рождения)
}

Н. М. Добровольский, Т. К. Иконникова, Е. С. Крупицын, В. Ю. Матвеев, Ю. В. Нестеренко, В. Н. Чубариков, М. В. Шамолин

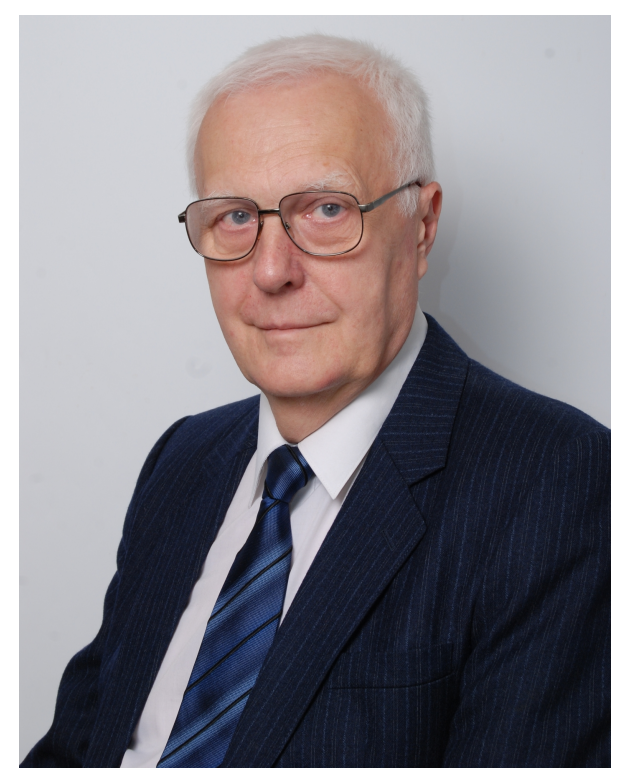

Владимир Григорьевич Чирский родился в Москве 30 июня 1949 года. Его родителями были Григорий Михайлович Чирский, начальник одного из управлений МПС и Елена Ивановна Фирстова, преподаватель английского языка на Госкурсах «Иняз». Его брат, Алексей Григорьевич Чирский, 1932 года рождения, был горным инженером. У него прекрасная семья, жена - Галина Владимировна, две дочери, Наталья и Ольга, четыре внука и внучка.

Владимир Григорьевич окончил среднюю школу №710 с серебряной медалью. Он учился на механико - математическом факультете МГУ имени М. В. Ломоносова и на третьем курсе начал изучать теорию чисел под руководством профессора А. Б. Шидловского, заведующего кафедрой теории чисел МГУ. По окончании факультета был рекомендован в аспирантуру отделения математики. К этому моменту им были опубликованы 4 статьи. С 1973 года начал работать на кафедре математического анализа сначала почасовиком, с 1975 года ассистентом кафедры. Защитил кандидатскую диссертацию в 1978 году и с 1983 года работал доцентом кафедры математического анализа. После защиты в 2000 году докторской диссертации работает профессором этой кафедры с 2001 года по настоящее время. С 1998 г. по 2006 г. работал заместителем декана механико-математического факультета по учебной работе. С 2007 года исполняет обязанности заведующего кафедрой теории чисел МПГУ, с 2006 года работает профессором в РАНХиГС. Кроме того, он является редактором раздела «Теория чисел» РЖК «Математика» ВИНИТИ РАН.

В. Г. Чирский преподавал и читал лекции на различных факультетах МГУ: механикоматематическом, химическом, геологическом, факультете психологии. Он является ответственным на кафедре за преподавание на химическом факультете. Он участвовал в издании 5 учебных пособий по математическому анализу и приложениям математики к задачам естествознания. Кроме того, он многие годы был старшим экзаменатором на вступительных 
экзаменах по математике на различных факультетах МГУ. Опыт этих экзаменов нашел отражение в 6 пособиях по элементарной математике. Под его руководством защищены две кандидатские диссертации, подготовлены к защите диссертации еще двух его учеников.

Научные интересы В. Г. Чирского относятся к теории трансцендентных чисел в $p$ - адических полях и прямых произведений этих полей. Используемый им метод представляет собой некоторую модификацию метода Зигеля-Шидловского в теории трансцендентных чисел. Изначально этот метод использовался для исследования арифметической природы $E$ - функций. Целая функция

$$
f(z)=\sum_{n=0}^{\infty} \frac{c_{n}}{n !} z^{n}
$$

называется $E$ - функцией, если выполнены следующие условия:

(i) Все коэффициенты $c_{n}$ принадлежат некоторому алгебраическому числовому полю $\mathbb{K}$ конечной степени $\varkappa$ над полем $\mathbb{Q}$ рациональных чисел.

(ii) Максимумы абсолютных величин алгебраически сопряжённых с числом $c_{n}$ чисел для любого $\varepsilon>0$ представляют собой $O\left(n^{\varepsilon n}\right), n \rightarrow \infty$. Для так называемой $E$ - функции в узком смысле это соотношение заменяется на $O\left(C^{n}\right), n \rightarrow \infty$ с некоторой постоянной $C>1$.

(iii) Существует последовательность натуральных чисел $d_{n}$ такая, что при $k=0,1, \ldots, n$ числа $d_{n} c_{k}$ принадлежат кольцу целых чисел $\mathbb{Z}_{\mathbb{K}}$ поля $\mathbb{K}$ и $d_{n}=O\left(n^{\varepsilon n}\right), n \rightarrow \infty$. (Для $E$ - функции в узком смысле это соотношение заменяется на $O\left(C^{n}\right), n \rightarrow \infty$ с некоторой постоянной $C>1$.)

В работах А. Б. Шидловского были получены основные теоремы о значениях $E$ - функций, получившие широкую известность. Сформулируем одну из них.

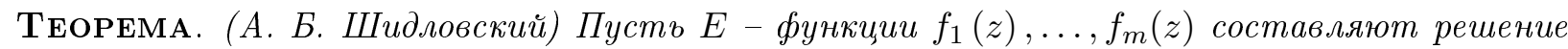
системы линейных дифференииалъных уравнений

$$
y_{i}^{\prime}=Q_{i, 0}(z)+\sum_{j=1}^{m} Q_{i, j}(z) y_{j}, i=1, \ldots, m
$$

с коэффициентами из поля $\mathbb{C}(z)$ рациональных функций от z и алгебраически независимъ над этим полем. Пусть $\alpha$ - алгебрачческое число, отличное от нуля и особых точек системы $(2)$. Тогда числа $f_{1}(\alpha), \ldots, f_{m}(\alpha)$ алгебраччески независимы, т.е. для любого отличного от нулевого многочлена $P\left(y_{1}, \ldots, y_{m}\right)$ выполняется неравенство

$$
P\left(f_{1}(\alpha), \ldots, f_{m}(\alpha)\right) \neq 0 .
$$

Эта теорема имеет многочисленные приложения к обобщённым гипергеометрическим $E$ функциям вида

$$
\sum_{n=0}^{\infty} \frac{\left(a_{1}\right)_{n} \ldots\left(a_{l}\right)_{n}}{\left(b_{1}\right)_{n} \ldots\left(b_{m}\right)_{n}}\left(\frac{z}{m-l}\right)^{(m-l) n}
$$

где все числа $a_{1}, \ldots, a_{l}, b_{1}, \ldots, b_{m}$ - рациональные, причём каждое из чисел $b_{1}, \ldots, b_{m}$ отлично от нуля и целых отрицательных чисел, число $m-l$ положительное, а символ $(c)_{n}$ определён равенствами: $(c)_{0}=1,(c)_{n}=c(c+1) \ldots(c+n-1), n=1,2, \ldots$ Практически полное решение 
задачи об условиях, при которых обобщённые гипергеометрические $E$ - функции алгебраически независимы над полем $\mathbb{C}(z)$, получено в работах ученика А. Б. Шидловского, профессора В. Х. Салихова.

В основополагающей работе К. Зигеля отмечена возможность применения этого метода к другому классу $G$ - функций. Ряд

$$
f(z)=\sum_{n=0}^{\infty} c_{n} z^{n}
$$

называется $G$ - функцией, если все коэффициенты $c_{n}$ принадлежат некоторому алгебраическому числовому полю $\mathbb{K}$ конечной степени $\varkappa$ над полем $\mathbb{Q}$ рациональных чисел и удовлетворяют тем же условиям, что и коэффициенты $E$ - функции в узком смысле. Исследуя арифметические свойства значений функций этого класса, Э. Бомбиери в 1981 году ввёл понятие глобального (алгебраического) соотношения. Пусть $P\left(y_{1}, \ldots, y_{m}\right)$ - отличный от нулевого многочлен с коэффициентами из поля $\mathbb{K}$ и пусть степенные ряды $f_{1}(z), \ldots, f_{m}(z)$ имеют коэффициенты из поля $\mathbb{K}$ и точка $\alpha \in \mathbb{K}$. Соотношение

$$
P\left(f_{1}(\alpha), \ldots, f_{m}(\alpha)\right)=0
$$

называется глобальным, если оно выполняется во всех полях $\mathbb{K}_{v}$, в которых сходятся все ряды $f_{1}(\alpha), \ldots, f_{m}(\alpha)$.

В работах В. Г. Чирского введён в рассмотрение новый класс степенных рядов, к которому удалось применить метод Зигеля-Шидловского для исследования глобальных соотношений, класс $F$-рядов. Естественным шагом стало исследование рядов вида

$$
f(z)=\sum_{n=0}^{\infty} c_{n} \cdot n ! \cdot z^{n} .
$$

Будем говорить, что этот ряд принадлежит классу $F\left(\mathbb{K}, C_{1}, C_{2}, C_{3}, q\right)$, если

(i) Все коэффициенты $c_{n}$ принадлежат некоторому алгебраическому числовому полю $\mathbb{K}$ конечной степени $\varkappa$ над полем $\mathbb{Q}$ рациональных чисел.

(ii) Максимумы абсолютных величин алгебраически сопряжённых с числом $c_{n}$ чисел представляют собой $O\left(e^{C_{1} n}\right), n \rightarrow \infty$ с некоторой постоянной $C_{1}$.

(iii) Существует последовательность натуральных чисел $d_{n}$ такая, что при $k=0,1, \ldots, n$ числа $d_{n} c_{k}$ принадлежат кольцу целых чисел $\mathbb{Z}_{\mathbb{K}}$ поля $\mathbb{K}$ и $d_{n}=q^{n} d_{0, n}, q \in \mathbb{N}$ а числа $d_{0, n}$ делятся только на простые числа $p$, не превосходящие $C_{2} n 0$ и для всех таких простых $p$ выполняется неравенство $\vartheta_{p}\left(d_{0, n}\right) \leqslant C_{3}\left(\log _{p} n+\frac{n}{p^{2}}\right)$.

Из свойства 3 следует, что если простое число $p$ не делит число $q$, то ряд (5), например, с рациональными коэффициентами $c_{n}$, имеет в поле $\mathbb{Q}_{p}$ радиус сходимости, равный $p^{\frac{1}{p-1}}>1$. Именно это обстоятельство позволяет применить к исследованию арифметических свойств значений таких рядов формулу произведения.

Известным примером $F$-ряда является ряд Эйлера $\sum_{n=0}^{\infty} n ! \cdot(-z)^{n}$. Разумеется, если ряд $(5)$ отличен от многочлена, он имеет в поле $\mathbb{C}$ нулевой радиус сходимости.

Легко заметить также, что если коэффициенты $c_{n}$ ряда (5) удовлетворяют перечисленным условиям, то ряд (4) с теми же коэффициентами $c_{n}$ является $G$ - функцией, а ряд (1) с теми же коэффициентами $c_{n}$ является $E$ - функцией в узком смысле.

Сформулированные ниже теоремы представляют собой некоторые аналоги основных теорем А. Б. Шидловского, справедливые для $F$ - рядов. 
ТеОрема 1 (Теорема 1.1 из (7) ). Пусть $F-$ рлды $f_{1}(z) \equiv 1, f_{2}(z), \ldots, f_{m}(z)$ линейно независимы над полем $\mathbb{K}(z)$ и составляют решение системы линейных дифференциальных уравнений

$$
y_{i}^{\prime}=Q_{i, 0}(z)+\sum_{j=1}^{m} Q_{i, j}(z) y_{j}, i=1, \ldots, m
$$

с коэффициентами из поля $\mathbb{C}(z)$ рациональных функций от z.

Пусть $\xi \neq 0, \xi \in \mathbb{K}$ - регулярная точка системы (6). Пусть

$$
L\left(y_{1}, \ldots, y_{m}\right)=h_{1} y_{1}+\ldots+h_{m} y_{m}-
$$

ненулевая линейная форма, $h_{i} \in \mathbb{Z}_{\mathbb{K}}, i=1, \ldots, m$. Пусть $H(L)=\max H\left(h_{i}\right)$.

Существует эффективно вычисляемая постоянная $H_{0}$ такая, что для любого

$$
H \geqslant \max \left(H_{0}, H(L)\right)
$$

существуют эффективно вычисляемая постоянная $C_{0}$, простое число $p$, удовлетворяющие неравенствам

$$
P_{l}(H)=l\left(\frac{\ln H}{\ln \ln H}\right) \leqslant p \leqslant u\left(\frac{\ln H}{\ln \ln H}\left(1+\frac{C_{0}}{\sqrt{\ln \ln H}}\right)\right)=P_{u}(H)
$$

и нормирование $v$ поля $\mathbb{K}$, продолжающее $p$ - адическое нормирование поля $\mathbb{Q}$ такие, что имеет место неравенство

$$
L(\xi)=L\left(f_{1}(\xi), \ldots, f_{m}(\xi)\right) \neq 0 .
$$

Замечание. Можно также для хотя бы одного $v$, продолжающее $p$ - адическое нормирование поля $\mathbb{Q}$ установить оценку

$$
|L(\xi)|_{v} \geqslant H^{-m-\frac{m+3+2 m C_{5}}{\sqrt{\ln \ln H}}} .
$$

Эта теорема допускает формулировку в несколько иных терминах. Рассмотрим множество точек вида:

$$
\left(x_{1}, x_{2}, \ldots, x_{n}, \ldots\right),
$$

образующих бесконечномерное пространство. Это пространство представляет собой прямое произведение полей $p$ - адических чисел. Именно, координата с номером $n$ этого вектора представляет собой $p_{n}$ - адическое число, где $p_{n}$ - простое число с номером $n$. Напомним, что $p$ - адические числа являются пополнением поля рациональных чисел по $p$ - адическому нормированию. Для рационального числа $a$ символ $|a|_{p}$ обозначает величину $p$ - адического нормирования, т.е. $|a|_{p}=p^{-\vartheta_{p}(a)}$, где, в свою очередь, символ $\vartheta_{p}(a)$ обозначает степень, в которой простое число $p$ входит в разложение числа $a$ на простые множители. Это прямое произведение имеет естественную структуру коммутативного кольца с единицей (и с делителями нуля). Его принято называть кольцом полиадических чисел. Элементы а кольца целых полиадических чисел имеют каноническое представление в виде

$$
\mathfrak{a}=\sum_{m=1}^{\infty} a_{m} m !, a_{m} \in \mathbb{N}, 0 \leqslant a_{m} \leqslant m .
$$

Степень, в которой простое число $p$ входит в разложение числа $n$ ! на простые множители равна $\frac{n-S_{n}}{p-1}$, где $S_{n}$ обозначает сумму цифр в $p$ - ичном разложении числа $n$. Следовательно, для любого $p$ при $n \rightarrow \infty$ выполняется соотношение $\left|a_{n} n !\right|_{p} \rightarrow 0$. Это - достаточное условие для 
того, чтобы ряд (7) сходился в поле $\mathbb{Q}_{p}$. Сумму этого ряда $(7)$ в поле $\mathbb{Q}_{p}$ обозначаем $\mathfrak{a}^{(p)}$. Как отмечено выше, полиадическое число $\mathfrak{a}$ можно рассматривать, как точку бесконечномерного пространства с координатами $\mathfrak{a}^{\left.(p)_{n}\right)}$, где $p_{n}-$ простое число с номером $n$. Следует отметить, что ряды вида $\sum_{n=0}^{\infty} a_{n} n !, a_{n} \in \mathbb{Q}$ могут сходиться не во всех полях $\mathbb{Q}_{p}$. В случае, когда такой ряд расходится лишь в конечном множестве полей $\mathbb{Q}_{p}$, мы говорим о почти полиадических числах и отождествляем их с элементами прямого произведения всех полей $\mathbb{Q}_{p}$, кроме упомянутого выше конечного множества.

Можно рассмотреть и прямое произведение полей $\mathbb{K}_{v}$, где $v$ продолжает $p$ - адическое нормирование поля $\mathbb{Q}$ на поле $\mathbb{K}$ алгебраических чисел конечной степени $\varkappa$. Если $v$ продолжает $p$ - адическое нормирование, то поле $\mathbb{K}_{v}$ представляет собой алгебраическое расширение поля $p$ - адических чисел $\mathbb{Q}_{p}$ степени $\varkappa_{v}$ и справедливо равенство $\sum_{v} \varkappa_{v}=\varkappa$, где суммирование производится по всем нормированиям $v$, продолжающим $p$ - адическое нормирование. Мы рассматриваем нормализованные нормирования, для которых $|p|_{v}=p^{-\frac{\varkappa v}{\varkappa}}$. Если нормирование $v$ продолжает обычную абсолютную величину, то ему соответствует алгебраически сопряжённое поле $\mathbb{K}^{(i)}$ и $\varkappa_{v}=1$, если $\mathbb{K}^{(i)}$ - подполе поля действительных чисел и $\varkappa_{v}=2$, если нет. Равенство $\sum_{v} \varkappa_{v}=\varkappa$ выполняется и в этом случае.

При этом мы будем говорить о $\mathbb{K}$ - полиадических и, соответственно, о $\mathbb{K}$ - почти полиадических числах. Для элемента $\mathfrak{a}$ этого прямого произведения обозначаем $\mathfrak{a}^{(v)}$ его координату в поле $\mathbb{K}_{v}$.

Если существует $P(x)$ - многочлен с рациональными коэффициентами, отличный от тождественного нуля такой, что $P(\mathfrak{a})=0$ (иными словами, $P\left(\mathfrak{a}^{(v)}\right)=0$ в каждом поле $\mathbb{K}_{v}$ этого прямого произведения), то говорим, что $\mathfrak{a}$ - алгебрачческий элемент. Если элемент $\mathfrak{a}$ не является алгебраическим, то его называют трансцендентным. Трансцендентность элемента означает, что для любого $P(x)$ - многочлена с рациональными коэффициентами, отличного от тождественного нуля, существует простое число $p$ и нормирование $v$ поля $\mathbb{K}$, продолжающее $p$ - адическое нормирование поля $\mathbb{Q}$ такие, что $P\left(\mathfrak{a}^{(v)}\right) \neq 0$ в поле $\mathbb{K}_{v}$. Назовём элемент $\mathfrak{a}$ бесконечно трансцендентным, если для любого $P(x)$ - многочлена с рациональными коэффициентами, отличного от тождественного нуля, существует бесконечное множество простых чисел $p$, для каждого из которых есть нормирование $v$ поля $\mathbb{K}$, продолжающее $p$ - адическое нормирование такое, что $P\left(\mathfrak{a}^{(v)}\right) \neq 0$ в поле $\mathbb{K}_{v}$. Элемент $\mathfrak{a}$ называется глобально трансцендентным, если для любого $P(x)$ - многочлена с рациональными коэффициентами, отличного от тождественного нуля, неравенство $P\left(\mathfrak{a}^{(v)}\right) \neq 0$ выполняется во всех полях $\mathbb{K}_{v}$ рассматриваемого прямого произведения.

Теорема 2. Пусть $F$ - рлды $f_{1}(z) \equiv 1, f_{2}(z), \ldots, f_{m}(z)$ составляют решение системы (6) и линейно независимы над полем $\mathbb{K}(z)$. Пусть $\xi \in \mathbb{K}$ - регулярная точка системь (6). Пусть

$$
L\left(y_{1}, \ldots, y_{m}\right)=h_{1} y_{1}+\ldots+h_{m} y_{m}--
$$

ненулевая линейная форма, $h_{i} \in \mathbb{Z}_{\mathbb{K}}, i=1, \ldots, m$. Тогда существует бесконечное множество простых чисел р и нормирований $v$ поля $\mathbb{K}$, продолжсающих $p$ - адическое нормирование поля $\mathbb{Q}$, таких, что в поле $\mathbb{K}_{v}$

$$
L\left(f_{1}(\xi), \ldots, f_{m}(\xi)\right) \neq 0 .
$$

Иными словами, рлды $f_{1}(\xi), \ldots, f_{m}(\xi)$ бесконечно линейно независимы.

ТеОрема 3. Пусть $F$ - рлды $f_{1}(z), f_{2}(z), \ldots, f_{m}(z)$ составляют решение системы (6) и алгебраически независимы над полем $\mathbb{K}(z)$. Пусть $\xi \in \mathbb{K}$ - регулярная точка системы (6). Пусть $P\left(y_{1}, \ldots, y_{m}\right)$ - ненулевой многочлен с коэффициентами из $\mathbb{Z}_{\mathbb{K}}$. Тогда существует бесконечное множество простых чисел $p$ и нормирований $v$ поля $\mathbb{K}$, продолжающих $p-$ адическое нормирование поля $\mathbb{Q}$, таких, что в поле $\mathbb{K}_{v}$

$$
P\left(f_{1}(\xi), \ldots, f_{m}(\xi)\right) \neq 0 .
$$


Иными словами, ряды $f_{1}(\xi), \ldots, f_{m}(\xi)$ бесконечно алгебраччески независимы

Сформулированные теоремы имеют приложения к обобщенным гипергеометрическим рядам. Символ Похгаммера определён равенствами

$$
(a)_{0}=1,(a)_{n}=a(a+1) \ldots(a+n-1), n \geqslant 1 .
$$

Для множества действительных чисел $S=\left\{\alpha_{1}, \ldots, \alpha_{r} ; \beta_{1}, \ldots, \beta_{s}\right\}$, относительно которых предполагаем, что числа $\beta_{1}, \ldots, \beta_{s}$ - не целые неположительные, принято обозначать

$$
{ }_{r} F_{s}\left(\begin{array}{ccc}
\alpha_{1} & \ldots & \alpha_{r} \\
\beta_{1} & \ldots & \beta_{s}
\end{array} ; z\right)=\sum_{n=0}^{\infty} \frac{\left(\alpha_{1}\right)_{n} \ldots\left(\alpha_{r}\right)_{n}}{\left(\beta_{1}\right)_{n} \ldots\left(\beta_{s}\right)_{n} n !} z^{n}-
$$

так называемый обобщённый гипергеометрический ряд. Можно рассматривать несколько более общие ряды вида

$$
\sum_{n=0}^{\infty} \frac{\left(\alpha_{1}\right)_{n} \ldots\left(\alpha_{r}\right)_{n}}{\left(\beta_{1}\right)_{n} \ldots\left(\beta_{s}\right)_{n}} z^{n}
$$

Ряды (8) и (9) относятся к так называемым рядам Жевре порядков $s+1-r$ и $s-r$, соответственно.

Если $r-s<0$, то рассматриваемый ряд (9) представляет собой целую функцию ( ряд (8) представляет целую функцию при $r-s \leqslant 0)$. К этим случаям относятся показательная функция, функции Бесселя, функции Куммера и большое количество других, важных в математике функций. При условии рациональности чисел $\alpha_{1}, \ldots, \alpha_{r} ; \beta_{1}, \ldots, \beta_{s}$ они входят в класс $E$ - функций Зигеля и к исследованию их значений применим известный метод ЗигеляШидловского в теории трансцендентных чисел [1]. Применению этого метода к гипергеометрическим $E$ - функциям посвящены работы В. Х. Салихова [8]-[10]. в которых получено близкое к полному решение проблемы. При $r-s=0$ ряд (9) имеет конечный радиус сходимости ( ряд (8) имеет конечный радиус сходимости при $r-s=-1$ ). $\mathrm{K}$ таким функциям относятся логарифмическая функция, гипергеометрическая функция Гаусса, многие алгебраические функции, неполные эллиптические интегралы и др. При условии рациональности чисел $\alpha_{1}, \ldots, \alpha_{r} ; \beta_{1}, \ldots, \beta_{s}$ они входят в класс $G$ - функций Зигеля и исследованию их посвящены работы А. И. Галочкина [11], Г. В. Чудновского [10], Э. Бомбьери [5], И. Андре [13] и др.

Если же $r-s>0$, то ряд (9), отличный от многочлена, имеет нулевой радиус сходимости в поле $\mathbb{C}$. (Ряд $(8)$ имеет нулевой радиус сходимости при $r-s \geqslant 0$ ). В этом случае получаются $F$ - ряды. Исследованию свойств таких рядов посвящены, например, работы [17]-[20]. В случае, когда среди параметров рассматриваемых гипергеометрических рядов содержатся иррациональные алгебраические числа, используя аппроксимации Эрмита-Паде, приведенные в [21], удается доказать бесконечную линейную независимость значений таких рядов в алгебраических точках [22].

Ещё одно направление исследований - вопросы алгебраической независимости элементов $\mathbb{C}_{p}$ над полем $\mathbb{Q}_{p}$. В работах В. Г. Чирского и П. Бундшу [34], [35], [46], [59] установлены теоремы, дающие достаточные условия алгебраической независимости над полем $\mathbb{Q}_{p}$ совокупностей рядов вида

$$
\sum_{n=0}^{\infty} a_{n} p^{r_{n}}
$$

где $a_{n}$ - единицы кольца $\mathbb{Z}_{p}$, а $r_{n}$ - возрастающая последовательность рациональных чисел. Рассмотрены также задачи об алгебраической независимости над полем $\mathbb{Q}_{p}$ значений аналитических функций в точках такого вида. 


\section{СПИСОК НАУЧНЫХ РАБОТ ЧИРСКОГО В. Г.}

1. Об арифметических свойствах значений некоторых функций.

Совместно с М. С. Нурмагомедовым // Вестн. Моск. Ун-та.- Сер.1, матем., механ.-1973.№1. -c.19- 26.

2. Об арифметических свойствах значений некоторых функций.

Совместно с М. С. Нурмагомедовым // Вестн. Моск. Ун-та.- Сер.1, матем., механ.-1973.№2. - c. $38-45$.

3. Об арифметических свойствах значений аналитических функций, связанных алгебраическими уравнениями над полем рациональных функций.// Матем. заметки.-1973.-т.14.вып.1. -с. 83 - 94 .

4. Об арифметических свойствах значений эллиптических интегралов. // Вестн. Моск. УНта.- Сер.1, матем., механ.-1973.-№5. -с.57 - 64 .

5. Об арифметических свойствах значений эллиптических интегралов. // Успехи матем. наук.-1977.-т.32.-№1(193). -с.211 - 212.

6. Об арифметических свойствах значений аналитических функций с алгебраическими коэффициентами рядов Тейлора. // Вестн. Моск. ун-та.- Сер.1, матем., механ.-1978.-№2. -c. $41-47$.

7. Об арифметических свойствах значений аналитических функций с алгебраическими иррациональными коэффициентами рядов Тейлора. // Вестн. Моск. ун-та.- Сер.1, матем., механ.-1978.-№3. -с.29 - 34.

8. Об арифметических свойствах значений гипергеометрических функций с иррациональными параметрами // Вестн. Моск. ун-та.- Сер.1, матем.,механ.-1978.-№3. -с.29 - 34.

9. Об арифметических свойствах значений эллиптических интегралов. // Вестн. Моск. ун-та.Сер.1, матем.,механ.-1979.-№1. -с.3 - 11.

10. Оценки линейных форм с алгебраическими коэффициентами. // Вестн. Моск. ун-та.Сер.1, матем.,механ.-1984.-№3. -с.32 - 35 .

11. Оценки многочленов от значений эллиптических интегралов.// Диофантовы приближения, ч.1, МГУ.-1985. -с.106 - 109.

12. О нетривиальных глобальных соотношениях. // Вестн. Моск. ун-та.- Сер.1, матем., механ. - 1989. - №5. -с.33 - 36

13. О глобальных соотношениях.// Матем. заметки.-1990.-т.48.-вып.2 -с.123 - 127.

14. Об алгебраических соотношениях в локальных полях. // Вестн. Моск. ун-та.- Сер.1, матем.,механ.-1990.-№3. -с.92 - 95 .

15. Глобальные соотношения и гипергеометрические ряды.// Успехи матем. наук.-1991.-т.46.вып.6(282). -с.221 - 222.

16. Об арифметических свойствах значений G-функций.// Вестн. Моск. ун-та. - Сер.1, матем., механ. - 1991. -№4. - с.84- 86 . 
17. Об арифметических свойствах значений некоторых функций. // Вестн. Моск. Ун-та.Сер.1, матем.,механ.-1992.-№4. -с.93 - 95.

18. Об алгебраических соотношениях в неархимедовски нормированных полях.// Функциональный анализ и прилож.-1992.-т.26.-вып.2. -с.41 - 50.

19. Об арифметических свойствах значений гипергеометрических функций. // Матем. заметки. -1992. -т.52. -выл.2. -с.125 - 131.

20. О рядах, алгебраически независимых во всех локальных полях.// Вестн. Моск. ун-та.Сер.1, матем.,механ.-1978.-№3. -с.29 - 34.

21. Оценки многочленов и линейных форм в прямых произведениях полей// Вестн.Моск.унта.-сер.1.матем. механ.-1994.-№4. -с.35 - 39.

22. Арифметические свойства значений гипергеометрических рядов.// Труды Матем. ин-та PAH.-1994.-т.207. -с.347 - 352.

23. О глобальных соотношениях для гипергеометрических рядов.// Труды Семин. Им. И.Г. Петровского.-1995.-№18. -с.204 - 212.

24. Об арифметических свойствах значений некоторых рядов.// Матем.записки.-1996.-т.2. c. $110-113$.

25. Об арифметических свойствах значений некоторых рядов.// Вестн. Моск. ун-та.- Сер.1, матем.,механ.-1997.-№2. -с.53 - 55 .

26. Об алгебраической независимости значений функций, удовлетворяющих системам функциональных уравнений.// Труды Матем. ин-та РАН.-1997.-т.218. -с.433 - 438.

27. Об арифметических свойствах значений некоторых функций.// Фундам. и прикл. матем.1998.-т.4.-№2. - -с.725 - 732 .

28. О линейных глобальных соотношениях.// Вестн. Моск. ун-та.- Сер.1, матем.,механ.-1998.№4. - - . $70-72$.

29. Линейная независимость $p$-адических значений некоторых $q$-базисных гипергеометрических рядов.// Фундам. и прикл.матем.-1999.-т.5,№2. -с.619 - 625.

30. Арифметические свойства некоторых p-адических чисел.// Вестн. Моск. ун-та.- Сер.1, матем.,механ.-1999.-№6. -с.16 - 19.

31. Приближения Эрмита-Паде для некоторых q-базисных гипергеометрических рядов.// Вестн. Моск. ун-та.- Сер.1, матем.,механ.-2000.-oㅡ.-с.7 - 11.

32. Арифметические свойства рядов в полях с неархимедовыми нормированиями. Москва.: Изд-во ЦПИ при мех.-мат. ф-те МГУ.-2000.-120стр.

33. Algebraic independence of elements from $C_{p}$ over $Q_{p}$. Совместно с P.Bundschuh //Arch.der Math.,79(2002), $345-352$.

34. Algebraic independence of elements from $C_{p}$ over $Q_{p}$, II, Acta Arithmetica,113.4(2004), 309 326, совместно с P.Bundschuh.

35. Effective estimates for global relations on Euler-type series, Ann. Fac.Sci. Toulouse, Vol XIII, №2, 2004, 241 - 260. Совместно с D.Bertrand, J.Yebbou. 
36. Метод Зигеля в $p$-адической области. Фундам. и прикл. Матем, 2005, том 11, №6, 221 230.

37. Обобщение понятия глобального соотношения. Записки научных семинаров ПОМИ, Том 322, 2005. Стр. $220-238$.

38. Рекуррентные соотношения для некоторых определителей. Фундаментальная и прикладная математика, 2010, т.16, №6, 173 - 175.

39. Алгебраическая независимость над $Q_{p}$ значений аналитических функций в точках из $C_{p}$. Вестник МГУ, сер 1.,матем.мех.,2010, №6, 25 - 27. Совм. с О.Ю.Баженовой.

40. Оценки линейных форм и многочленов от совокупностей полиадических чисел. Чебышевский сборник, 2011, №4.

41. Оценки многочленов от некоторых $g$-адических чисел. Вестник МГУ, сер.1, матем,механ, 2012, №2, 58 - 59. Совм. С Е.С.Крупицыным.

42. Арифметические свойства некоторых полиадических рядов. Вестник МГУ, сер.1, матем,ммехан, 2012, №5

43. Recurrent relations for certain determinants.в журнале Journal of Mathematical Sciences, издательство Plenum Publishers (United States), 2012, том 182, №4, с. 565 - 566 DOI

44. О некоторых свойствах полиадических разложений (совм. с В. Ю. Матвеевым). // Чебышевский сборник. 2013. Т. 14, №2, с. 163 - 171.

45. Values of Analytic Functions at points of $C_{p}$. Russian Journal of Mathematical Physics, издательство Maik Nauka/Interperiodica Publishing (Russian Federation), 2013.-том 20, №2, c. $149-154$

46. On the Arithmetic Properties of Polyadic Integers. International Mathematical Forum, том 8, №37, c. 1793 - 1796

47. Об арифметических свойствах обобщённых гипергеометрических рядов с иррациональными параметрами. Известия РАН. Серия математическая, 2014.том 78, № 6, с. 193 - 210

48. Арифметические свойства полиадических рядов с периодическими коэффициентами. Доклады Академии наук, издательство Наука (М.), 2014. том 459, №6, с. 677 - 679

49. On the arithmetic properties of generalized hypergeometric series with irrational parameters. Izvestiya. Mathematics, издательство American Mathematical Society(United States), 2014. том 78 , №6, с. $1244-1260$

50. Arithmetic properties of polyadic series with periodic coefficients. Doklady Mathematics, издательство Maik Nauka/Interperiodica Publishing (Russian Federation), 2014.-том 90, № 3, c. $766-768$

51. Об одном подходе к преобразованию периодических последовательностей. совм. с Нестеренко А.Ю.Дискретная математика, издательство Наука(М.), 2015.-том 27, №4, с. 150 157

52. Арифметические свойства целых полиадических чисел. Чебышевский сборник, издательство Федеральное государственное бюджетное образовательное учреждение высшего профессионального образования "Тульский государственный педагогический университет им. Л.Н. Толстого"(Тула), 2015.- том 16, №1, с. $254-264$ 
53. Estimating polynomials over $Z_{p}$ at points from $C_{p}$. (совм. с Bundschuh P.). Moscow Journal of Combinatorics and Number Theory, 2015. том 5, №1-2, с. 14-20

54. Arithmetic properties of Euler series. Moscow University Mathematics Bulletin, 2015. том 70, №1, c. $41-43$

55. О преобразованиях периодических последовательностей. Чебышевский сборник, издательство Федеральное государственное бюджетное образовательное учреждение высшего профессионального образования "Тульский государственный педагогический университет им. Л.Н. Толстого"(Тула), 2016. том 17, №3, с. $180-185$

56. Представление натуральных чисел слагаемыми определённого вида. Современные проблемы математики, 2016. -№24, с. 81 - 84

57. Периодические и непериодические конечные последовательности. Чебышевский сборник, издательство Федеральное государственное бюджетное образовательное учреждение высшего профессионального образования "Тульский государственный педагогический университет им. Л.Н. Толстого"(Тула), 2017. -том 18, №2, с. 275 - 278

58. Арифметические свойства полиадических рядов с периодическими коэффициентами. Известия РАН. Серия математическая, 2017.-том 81, № выпуск 2, с. 215 - 232

59. Topical problems of the theory of Transcendental numbers: Developments of approaches to tyeir solutions in the works of Yu.V. Nesterenko. Russian Journal of Mathematical Physics, издательство Maik Nauka/Interperiodica Publishing (Russian Federation), 2017. том 24, №2, c. $153-171$

60. Representation of positive integers by summands of a certain form. Proceedings of the Steklov Institute of Mathematics, издательство Springer Verlag (Germany),2017.- том 298, №1, c. $70-73$

61. Arithmetic properties of polyadic series with periodic coefficients. Izvestiya. Mathematics, uздательство American Mathematical Society (United States), 2017. -том 81, №2, с. 444 - 461

62. An approach to the transformation of periodic sequences. (coвm.c Nesterenko A.Yu). Discrete Mathematics and Applications, издательство VS P (Netherlands), 2017. том 27, №1, с. 1 - 6.

63. Арифметические свойства обобщенных гипергеометрических $F$-рядов. Доклады академии наук, 2018, том 483, №3. с. 252 - 254 .

64. Arithmetic Properties of Generalized Hypergeometric F-series. Doklady Mathematics. 2018. Vol.98.-No 3. -589 - 591.

\section{Книги по элементарной математике}

65. Уравнения элементарной математики. Совместно с Е.Т. Шавгулидзе. Москва, «Наука», 1992.

66. Методы решения задач по алгебре. Совместно с С.В.Кравцевым, Ю.Н.Макаровым, В.Ф.Максимовым, М.И.Нараленковым. Москва, «Экзамен», 2001.

67. Математика. Письменный экзамен. М. «Экзамен», 2006.511 стр. Совместно с А.И.Козко и Ю.Н. Макаровым. 
68. Задачи с параметрами и другие сложные задачи. М.МЦНМО, 2007, 2008, 296 стр. Совместно с А.И.Козко.

69. Математика. Задача С5. М.МЦНМО,2010-2013. Совместно с А.И.Козко, В.С. Панфёровым, И.Н. Сергеевым.

70. Задачи с параметрами, сложные и нестандартные задачи. М.МЦНМО, 2016, 229 стр. Совместно с А.И.Козко, В.С. Панфёровым, И.Н. Сергеевым.

\section{Учебники и учебные пособия}

71. Математический анализ. М.: «Академия», 2013,336стр. Совместно с В.И. Гавриловым и Ю.Н. Макаровым.

72. Математические методы решения химических задач. М.: «Академия», 2013,367стр. Совместно с А.И.Козко, С.В. Кравцевым, Н.Б. Малышевой, Е.С. Соболевой, А.В. Субботиным, Г.М. Фатеевой.

73. Дифференциальные уравнения и математические модели химических задач. М: МЦНМО, 2019, 168 стр. Совместно с А.И.Козко.

74. Математический анализ и инструментальные методы решения задач. Книга 1: учебник / В. Г. Чирский, К. Ю. Шилин. - М.: Издательский дом «Дело» РАНХиГС, 2019. 463 с. - (Учебники Президентской академии).

75 Математический анализ и инструментальные методы решения задач. Книга 2: учебник / В. Г. Чирский, К. Ю. Шилин. - М.: Издательский дом «Дело» РАНХиГС, 2019. 258 с. - (Учебники Президентской академии).

Получено 18.03.2019 г.

Принято в печать 12.07.2019 г. 